\title{
Co-evolution of Microstructure and Magnetic Properties in Magnetically Aligned MnBi-Bi Composites
}

\author{
Stephen R. Boona ${ }^{1 *}$, Brandi Wooten ${ }^{2}$, Koen Vandaele ${ }^{3}$ and Joseph P. Heremans ${ }^{2,3}$ \\ 1. Center for Electron Microscopy and Analysis, The Ohio State University, Columbus, OH, USA. \\ 2. Department of Materials Science and Engineering, The Ohio State University, Columbus, OH, USA. \\ 3. Department of Mechanical \& Aerospace Engineering, The Ohio State University, Columbus, OH, \\ USA. \\ * Corresponding author: boona.1@osu.edu
}

Microstructural characterization workflows suitable for analysis of magnetic composites have become increasingly important due to growing interest in the development of such materials for applications ranging from rare-earth free permanent magnets to energy conversion and storage devices [1-3]. Here, we discuss the use of correlative imaging, spectroscopy, and diffraction techniques in a scanning electron microscope (SEM) to track the microstructural evolution of MnBi-Bi composites synthesized via melt quenching and magnetic field annealing, and then correlate these microstructural changes with the materials' magnetic properties.

$\mathrm{MnBi}$ is a strong room temperature ferromagnet with very favorable properties in which the large Mn magnetic moments are oriented along the c-axis of the NiAs-type hexagonal structure $[1,4]$. Previous reports indicate the $\mathrm{Mn}-\mathrm{Bi}$ phase diagram can be exploited to produce elongated $\mathrm{MnBi}$ grains preferentially orientated within a Bi single crystal matrix, producing a material with strongly anisotropic magnetic susceptibility [5]. To date, however, there has been no systematic study reported that tracks how this anisotropy changes with composition in samples that have been quenched and annealed.

To address this gap, we have synthesized a series of MnBi-Bi composite materials and studied their microstructure using a ThermoFisher Apreo LoVac SEM. Secondary and backscatter electron images were produced using the instrument's in column Trinity detectors, while the samples' compositions were studied via energy dispersive spectroscopy (EDS) using an EDAX Pegasus $30 \mathrm{~mm}^{2}$ detector, which was correlated with grain size and orientation information obtained via electron backscatter diffraction (EBSD) data collected with an EDAX Hikari camera analyzed with OIM and NPAR software. The samples' magnetic susceptibilities were measured separately with a Quantum Design Magnetic Property Measurement System using a Superconducting Quantum Interference Device (SQUID).

The extremely soft nature of the primary Bi matrix (Mohs hardness of 2.25), combined with the tendency of $\mathrm{MnBi}$ to readily decompose into $\mathrm{MnO}_{2}$ and $\mathrm{Bi}$ metal, each presented significant sample preparation challenges for EBSD. Despite these challenges, we were able to characterize the unique microstructural features of each sample and successfully correlate these changes with other properties.

Composites with low Mn content (1\%-2\% Mn) primarily showed segregation of the MnBi particles to the boundaries between much larger Bi grains, with the formation of only some very small, looselyaligned needle-type structures. Materials with intermediate Mn content (10\%-20\%) were observed to be largely inhomogeneous, containing many small, needle-like MnBi particles dispersed among Bi grains in addition to several much larger elongated structures. Samples containing large amounts of $\mathrm{MnBi}$ (20\% and above) displayed very large, elongated, and interconnected MnBi grains, as well as numerous 
large, 10-500 micron voids present throughout the cylindrical ingots, but especially near the outer rim. The 50\% MnBi sample contained a region of unreacted Mn metal near the center, indicating insufficient cooling rates during quenching. EBSD results provided insights about the alignment of MnBi structures and preferred orientation of neighboring $\mathrm{Bi}$ grains. These observations are consistent with magnetization measurements, which indicate the overall magnetic easy-axis of the composites is parallel to the direction of applied field, and that the remnant anisotropy ratio increases with increasing Mn content.

This work demonstrates a successful workflow for preparation of extremely soft composite materials for EBSD, as well as direct correlation of anisotropic magnetic properties with anisotropic microstructure. These results indicate that preferential alignment of magnetic grains (and therefore large anisotropy of the overall magnetic properties) can be achieved in composites synthesized by melt quenching followed later by in-field annealing $[5,6]$.

\section{References:}

[1] J Cui et al., Acta Materialia 158 (2018), p. 118.

[2] S Boona et al., Nature Commun. 7 (2016), p. 13714.

[3] K Vandaele et al., Mater Today Phys 1 (2017), p. 39.

[4] M A McGuire et al., Phys Rev B 90 (2014), p. 174425.

[5] J M Noothoven van Goor and H Zijlstra, J App Phys 39 (1968), p. 5471.

[6] The authors acknowledge funding from the OSU Center for Emergent Materials, an NSF MRSEC, NSF DMR 1420451, as well as direct support from the OSU Center for Electron Microscopy and Analysis. Dr. S Boona is grateful for sample preparation and EBSD advice from Dr. S Kuhr, B Welk, Z Kloenne, H Whitt, and N Taylor.

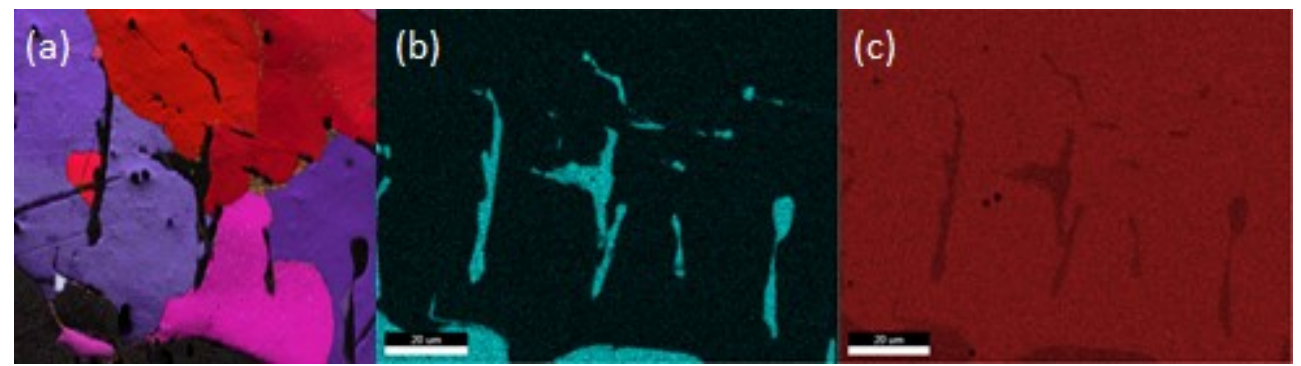

Figure 1. (a) EBSD inverse pole figure of $\mathrm{Bi}$ grains in $20 \% \mathrm{MnBi}$ sample; EDS map of $\mathrm{Mn}$ (b) and $\mathrm{Bi}$ (c) within same viewing area.
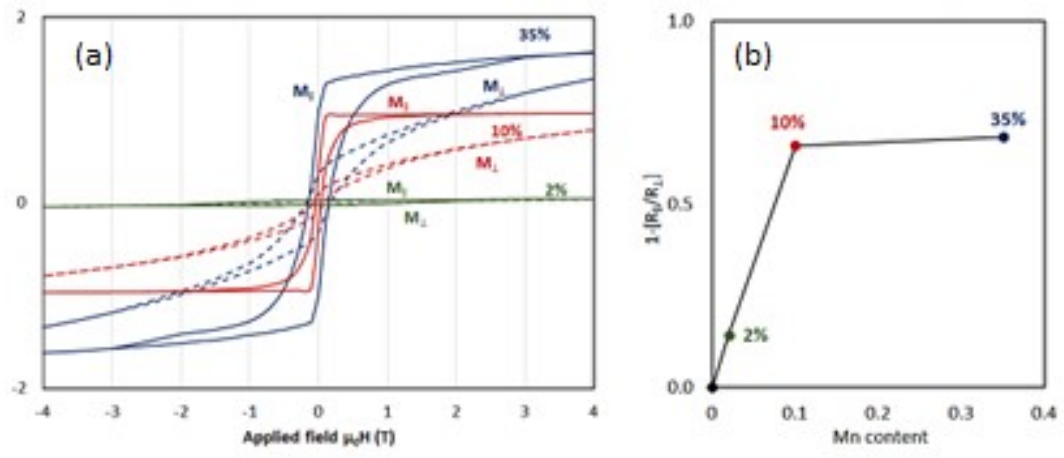

Figure 2. (a) Magnetization (arbitrary units) vs applied magnetic field for three MnBi-Bi composite samples with the labeled Mn content with $\mathrm{MnBi}$ grains oriented perpendicular and parallel to applied field, as indicated; (b) Remnant magnetic anisotropy ratio of the same three samples. 